\title{
Simulation of Aircraft Images for Airport Surface Traffic Control
}

\author{
Fausto MARTI, Maurizio NALDI \\ Centro Vito Volterra \\ TorVergata University of Rome
}

\begin{abstract}
An Air Surface Traffic Control (ASTC) system needs an advanced and integrated surveillance function based on radar sensors for noncooperative targets. A new generation of Surface Movement Radars is currently being developed that requires the use of tools like radar-image simulators, able to test the performances of such radars. A high resolution radar-image simulator that meets the ASTC requirements is described in this paper.
\end{abstract}

\section{The environment and the application.}

Greater safety and efficiency of air transport require an enhanced Air Traffic Management based on a co-ordination among air route traffic control, approach control and surface movement control. In particular, the subsystems, the tools and the operational procedures used for Air Surface Traffic Control (ASTC) have to be integrated, and a better coordination with Air Traffic Control and Management has to be implemented.

ASTC requires an efficient surveillance function to ensure greater safety. There is a growing need for information about precise position and identity of a target, as well as for other target information, which must be reliable and obtained at high rate.

Surveillance is an important function that provides a detailed picture of all the ground traffic (aircraft and vehicles) on the surface area of an airport. The environment of ASTC [2] is divided into a Movement area and an Apron area. The former includes approaches, runways, taxiways and all intersections between taxiways and runways. The latter is a defined area (on a land aerodrome) used to accommodate aircraft to load or unload passengers, mail and for refuelling, parking and maintenance purposes.

The airport environment is characterized by the presence of many tens of aircraft (with dimensions ranging from 10 to $70 \mathrm{~m}$ ), hundreds of various types of vehicles, (from small cars to large trucks), and numerous obstacles. The number of simultaneously moving targets to be tracked is of the order of five hundreds or more in large airports. Some targets can be cooperative, i.e. carrying equipment for detection and location. Therefore, in the future, the surveillance for ASTC will integrate the use of different types of sensors with data fusion.

A Surface Movement Radar (SMR) is of paramount importance, as it is capable of detecting and locating non-cooperative targets such as obstacles, intruders, etc. The main reason why surveillance is so important for safety is the need for detection of 
runway incursions and detection of not allowed and dangerous ground movements, such as wrong directions on runways and taxiways. An advanced ASTC system covers a whole aerodrome surface, and provides information about the detection, tracking, classification and orientation of aircraft and vehicles. These functions are used for guidance and control as well as for the detection and management of dangerous movements.

The main characteristics and functions of an SMR to be considered in image simulation can be summarized as follows:

- high tracking precision and class labelling (i.e. aircraft classification depending on dimensions);

- image processing to extract information such as target centroid, target dimensions and target orientation, necessary to implement the tracking algorithms for a large target, like an aircraft, and to improve the performance of the safety logic, which provides alarms or warnings when conflicts are identified on the basis of safety rules.

The image-processing techniques (Moment of Inertia, Hough Transform, CrossCorrelation) that can be applied to digitized radar images of aircraft taxiing on an airport surface are described in [3].

Tests of tracking algorithms and of image-processing techniques require appropriate images resolution and grey-dynamics range, in agreement with the resolution (higher than $3 \mathrm{~m} \times 6 \mathrm{~m}$ ) and the dynamics range (about $80 \mathrm{~dB}$ before logarithmic conversion) of the SMR used. In conclusion, a radar-image simulator should provide images of aircraft of an airport surface that are in agreement with the electro-magnetic characteristics of the targets, with the characteristics of next-generation sensors and with the target-sensor positions. The main characteristics of the sensor considered (a millimeter wave SMR) are described in [1] and in [4].

\section{Description of the Simulator}

This paper describes a simulator of aircraft images for Airport Surface Traffic Control. The simulator aims to test and evaluate:

- radar image processing algorithms;

- plot-extraction algorithms;

- track-while-scan algorithms for extended targets;

- sensor-data fusion.

The motivation for the development of the simulator is the lack of equivalent tools. In fact, some aircraft image simulators exist (e.g., the Radar Imagery Simulator, RIG), by the Technology Service Corporation), but such tools exhibit the following limitations:

a) the electromagnetic model of backscattering is valid up to $16 \mathrm{GHz}$ only, not for the millimeter-wave range;

b) such tools are not easy to integrate with other software for the purpose of research and study.

The software of the proposed SMR implements a simple aircraft electro-magnetic model suitable for the millimeter band $(95 \mathrm{GHz})$. The goal is to provide images of an 
aircraft like the new images obtained by a new-generation SMR image. The radar simulation is suitable for a monostatic radar, but simple modifications can allow the simulation of a bistatic radar.

It includes computation and graphics routines in $\mathrm{C}$ language and utilizes the $\mathrm{X}$ Window ToolKit. A simple block diagram is shown in Fig. 1

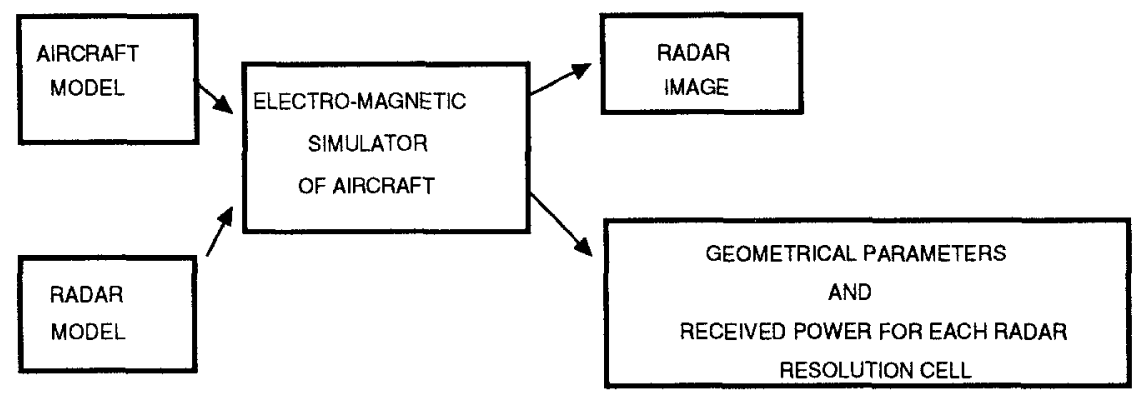

Figure 1 Block Diagram of the simulator.

\subsection{Radar Imaging for ASTC}

Radar imaging differs from optical (IR, visible, etc.) imaging in two main aspects:

a) Radiation coherence that causes statistical fluctuations (speckle) in time and from pixel to pixel; averaging of independent samples (in terms of time, frequency or polarization) is needed when speckle reduction is required.

b) A polar reference system (range and azimuth coordinates) that makes cross-range resolution vary linearly with the range; in our application, the cross-range resolution is between $0.5 \mathrm{~m}$ (at $150 \mathrm{~m}$ from the radar) and $5.2 \mathrm{~m}$ (at $1500 \mathrm{~m}$ from the radar), and the range resolution is $3 \mathrm{~m}$.

\subsection{The Radar Model}

The features of the radar sensor $[1,2]$ are used to develop the radar model: frequency, antenna rotation speed (r.p.m.), range resolution, azimuth resolution, pulse repetition frequency (PRF), and position of the radar. They are stored in a first data- file at the input to the simulator. Other parameters are derived during simulation.

The transmission and reception of each pulse (or, to reduce the computation load, of 1 out of $\mathrm{N}$ pulses) are simulated. The model for simulating the radar while the antenna is rotating is that of a linear system whose impulse response is a sequence of pulses received from a point-like scatterer. Therefore, for each range interval, the imaging process, while the antenna is scanning, corresponds to a convolution of the target reflectivity function over the radar impulse response.

\subsection{The Aircraft Model}

The second file at the input describes an aircraft (an air carrier or a general aircraft like the Boeing, Airbus, MD, DC and Cesna airplanes). It provides information about its real shapes and dimensions (from AutoCAD files). In order to build up a 
reasonably simple electromagnetic model of the aircraft, the aircraft surfaces are described by spherical, conical, cylindrical, or flat elements. The aircraft parts that may result in double or triple reflections (e.g., the connections between the wings and the fuselage, the tail wings / rudder, the engine intake inlets) are described by dihedra or trihedra. Therefore, the file is a 3D representation of the aircraft obtained by combining the following geometrical primitives: a cylinder, a truncated cone, a parallelepiped, a dihedron, and a trihedron (see Fig. 2).

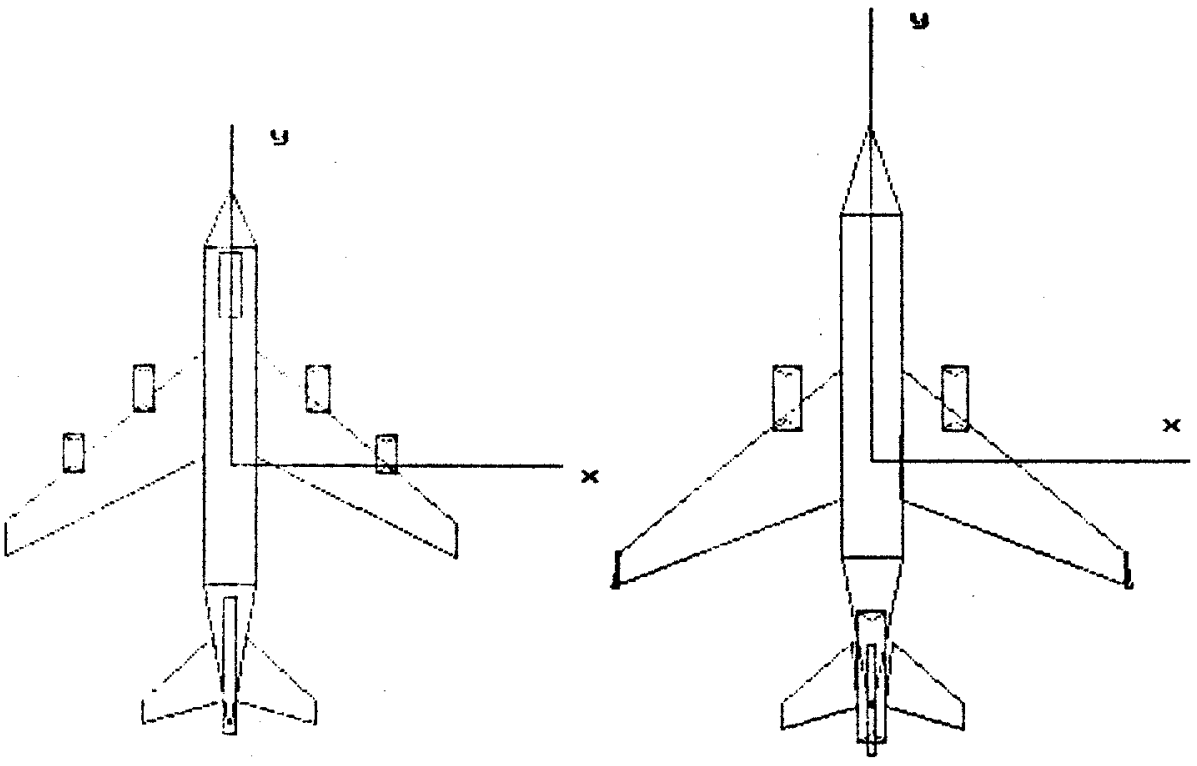

Figure 2 - Section representations of the $B 747$ and MDII Aircraft

Each geometrical 3D component is described by the coordinates of this component with respect to the reference system of the aircraft. The aircraft (target) is a large and complex object, and it is important that its representation should be easy to the user. The computer code of the simulator automatically exploits the representation of the target and the radar characteristics to identify which components are visible to the radar (at any given aspect angle), by using ray-tracing techniques. In order to describe the interaction between the radiation and the aircraft surface, the latter is modelled as a set of contiguous flat elements (facets) whose dimensions are a tradeoff between the need for minimizing the differences with respect to the model surface (i.e., the cylinder, the truncated cone, etc.) and the need for using facet dimensions that are much larger than the wavelength (equal to about $3.3 \mathrm{~mm}$ in our application) in order to apply Optical Physics. Typical facet dimensions are about $0.3 \mathrm{~m}$, or larger. This information, combined with the aspect angle and with optics-based scattering models, provides the Radar Cross Section, i.e. the backscattering capability (see below) of each scattering center and the power in each radar resolution cell. 
For such a target, the main problems lie in representing the model components and in establishing which ones are visible.

The steps to define and build up the aircraft model are the following:

1. analysis of the geometrical structure and of its dimensions;

2. splitting of the aircraft into elementary geometrical 3D components, such as a cylinder, a flat plate, a truncated cone, a parallelepiped, a dihedron, and a trihedron;

3. representation of each component element, with the related scattering law and the related position;

4. definitions of easy data structure files in order not to cause errors on the complex model.

\subsection{Output format}

The image provided by the simulator measures $64 \times 64$ pixels for an area of $128 \mathrm{~m} \mathrm{x}$ $128 \mathrm{~m}$ (i.e., larger than an aircraft). The resolution is $2 \times 2 \mathrm{~m} /$ pixel. The output is consistent with the characteristics of the SMR graphic display. In particular, the SMR uses a dedicated window of the work-space to display a surface area of the airport with the aircraft. The output file includes data on the radar position and on the signal power returned from each resolution cell.

\section{RCS calculation methods}

The Radar Cross Section of a target is a measure of the strength of the scattered field for a given illumination. It can be defined as [5]:

$$
R C S=\sigma(\theta, \varphi)=4 \pi \lim _{R \rightarrow \infty} R^{2} \frac{\left|E^{S}\right|^{2}}{\left|E^{\prime}\right|^{2}}
$$

where: $\quad \theta, \varphi$ are the azimuth and elevation angles;

$\mathbf{R}$ is the distance between the radar and the target;

$\mathrm{E}^{\mathbf{S}}$ is the scattered electric field;

$\mathbf{E}^{\mathbf{I}}$ is the incident electric field.

A technique that can be applied to a large target is to split it into a collection of smaller scatterers (flat plates). The returns from these can be coherently combined so as to give the total RCS, i.e., the echo of each scattering centre is a complex number that represents both the magnitude and the phase of the return,

$$
V_{n}(\theta, \varphi)=A_{n}(\theta, \varphi) e^{i \psi_{n}(\theta, \varphi)}
$$

with

$$
\Psi_{n}(\theta, \varphi)=\frac{4 \pi}{\lambda} R_{n}(\theta, \varphi)
$$


The total RCS of each resolution cell is:

$$
\operatorname{RCS}_{\text {TOT }}=\left|\sum_{1} v_{i}(\theta, \varphi)\right|^{2}
$$

The model based on physical optics theory is one of the simplest and most robust models for high-frequency scattering. Results on the RCSs of flat and curved surfaces, like cylinders, are available. As an example, the resulting RCS of a flat plate is:

$\mathrm{RCS}_{\text {flat }}=\frac{4 \pi}{\lambda^{2}}\left(\mathrm{~L}_{\mathrm{a}} \mathrm{L}_{\mathrm{b}} \cos (\theta) \cos (\phi)\right)^{2}\left(\frac{\sin \left(\mathrm{kL}_{\mathrm{a}} \sin \theta\right)}{\mathrm{kL}_{\mathrm{a}} \sin \theta}\right)^{2}\left(\frac{\sin \left(\mathrm{kL}_{\mathrm{b}} \sin \varphi\right)}{\mathrm{kL}_{\mathrm{b}} \sin \varphi}\right)^{2}$

where: $\quad$ k: $\quad$ wave number $=2 \pi / \lambda$;

$\theta: \quad$ incidence angle on the horizontal plane;

$\varphi: \quad$ incidence angle on the vertical plane;

$\mathbf{L}_{\mathbf{a}}:$ dimensions of the flat plate on the horizontal plane;

$\mathbf{L}_{\mathbf{b}}$ : dimension of the flat plate on the vertical plane;

$\lambda$ : wave length.

\section{Functional structure of the simulator of aircraft images.}

Figure 3 describes the fundamental simulation functions. The block diagram (bottom) describes them in more detail.

a) The first step is to define the aircraft with its shape and dimensions, its 3D elementary geometrical components, the type of scattering law for each elementary (a few wavelengths in size) subscatterer (flat plate) in the reference system of the aircraft. This kind of representation is independent of the position of the aircraft with respect to the radar.

b) The second step includes an analysis of the elementary flat plates that are visible to the radar, as a function of the positions of the target and of the radar, and then the calculation of the complex echo $\mathrm{V}_{n}(\theta, \varphi)$.

c) Finally, for each position of the radar, the total RCS, (Eq. (4)), and the power of the echo signal for each range and azimuth resolution cell, are calculated. This is accomplished with respect to the reference system of the radar.

The echo power is obtained by:

(l) computation of the RCS of each elementary scatterer inside a resolution cell (Eq. (5) for flat scatterers);

(II) computation of the amplitude of each elementary scattered field as the square root of the RCS, weighted by the 1-way antenna power gain, and computation of its phase by Eq. (3);

(III) sum of all the above computations for each resolution cell to get the total echo power for the assumed geometry. 
To simulate the antenna revolution, steps (II) and (III) correspond to a convolution that is made over the reference system of the radar.
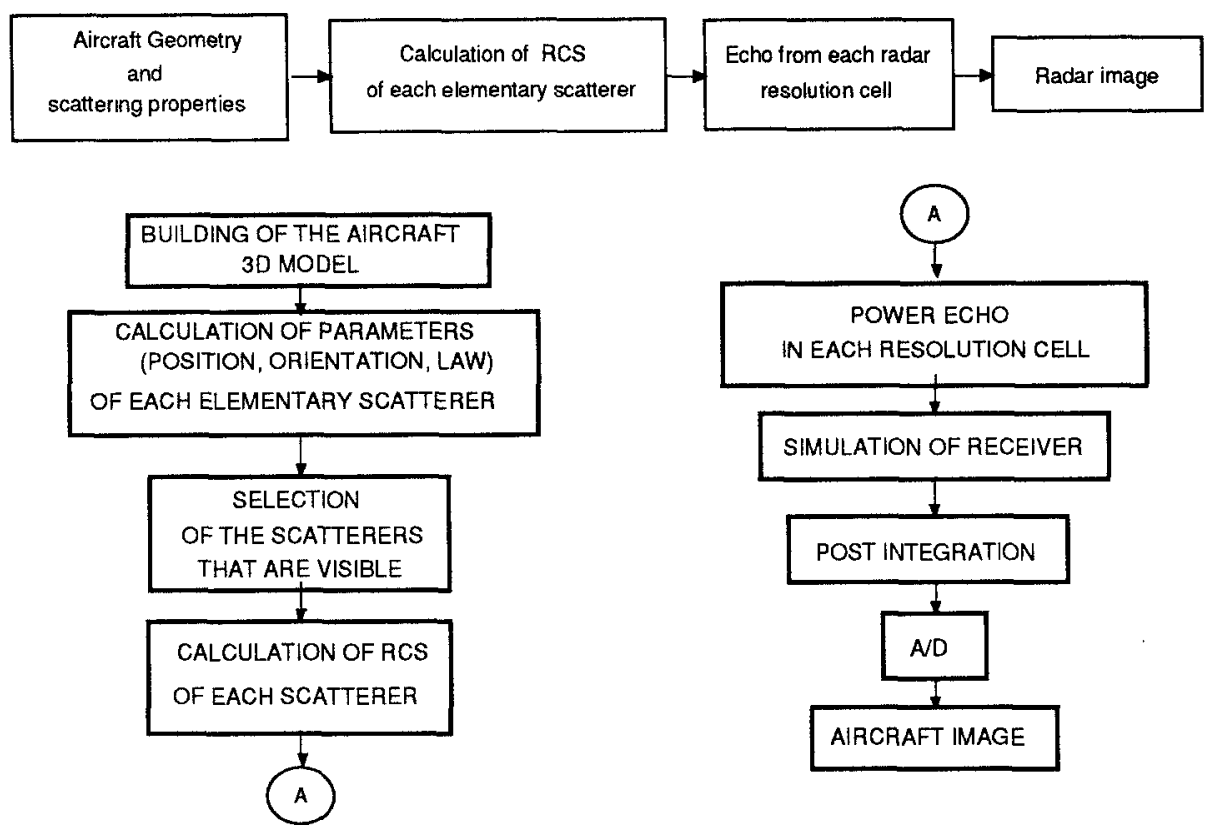

Figure 3 - Fundamental simulation functions.

\subsection{Results and computational complexity}

For a large aircraft, like the B747 (70 m long and $65 \mathrm{~m}$ wide), the number of scattering centres (i.e., the number of facets that form the elementary geometrical 3D components of the aircraft model) is about 70000 . The fuselage, the prow, the engines and the tails of the aircraft are divided into flat plates of typical dimensions of $0.3 \mathrm{~m}$.

The 3D model of this aircraft is recorded into a file whose size is about 3.5 Mbytes, as, for each scatterer, it is necessary to record its position with respect to the reference system of the aircraft, the type of scatterer (i.e., the type of scattering law) and the matrix coefficients to pass from the reference system of the aircraft to a local coordinate frame of a facet (local reference system). For this local reference system, the condition of visibility of this scatterer center with respect to the radar sensor is evaluated; this is done by evaluating the possible presence of an intersection between a generic 3D element of the model and the ray that goes from the radar to the scatterer center.

The software includes graphics and independent computation functions in C language and utilizes the Xwindows ToolKit to provide and to manage a Graphical User Interface (GUI): windows suitable for providing the output image, the output data and the description of the input data. The size of the executable software is 
about 150 Kbytes and the CPU time to obtain an image is less than one second (SUN SPARK CLASSIC 10). Finally, the number of multiplications is about 500 and the number of calls to the $\mathrm{C}$ functions (like sin and cos functions) is about 100 for each elementary scattering center. $90 \%$ of the total computation load is due to the resolution of the masking problem, i.e., the calculation of the scattering centers that are visible.

Figure 4 shows an image of the aircraft $A 310-200$ as a simulation output. In this first version, the algorithm used to resolve the masking problem is simplified, so the number of scattering centers is reduced.

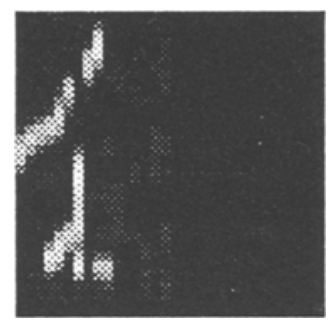

Figure 4 -Aircraft image provided by the simulation

\section{References}

1 G. Galati, M. Ferri, and F.Marti, Advanced Radar Techniques for the Air Transport System: the Surface Movement Miniradar Concept, IEEE National Telesystems Conference, S.Diego, May 1994.

2 G. Galati, M. Ferri, and F.Marti, Distributed advanced surveillance for SMGCS, ECAC-APATSI-EC Workshop on SMGCS, Frankfurt, April 1994.

3 G. L. Foresti, M. Frassinetti, G. Galati, F. Marti, P.F. Pellegrini, C. Regazzoni, Image Processing Applications to Airport Surface Movements Radar Surveillance and Tracking, IECON' 94, Bologna, September, 1994.

4 G. Galati, M. Ferri, P. Mariano, and F. Marti, Advanced Integrated Architecture for Ground Movements Surveillance, IEEE International Radar Conference, Alexandria, Virginia, May 1995.

5 M.I. Skolnik, Introduction To Radar System, McGraw-Hill International Ed., 1993. 